\title{
Obstructive sleep apnea basics
}

\section{ABSTRACT}

Obstructive sleep apnea (OSA) is a condition caused by repeated episodes of upper airway collapse and obstruction during sleep associated with arousal from sleep with or without oxygen desaturation. OSA is a highly prevalent condition, particularly in individuals with established risk factors and comorbid conditions. Screening for OSA includes a sleep history, review of symptoms, and physical examination, the results of which can identify patients that need testing for OSA. The polysomnogram or home sleep apnea test results aid in the diagnosis of OSA and its severity.

\section{KEY POINTS}

OSA is characterized by repeated episodes of complete or partial obstruction of the airway during sleep.

The prevalence of OSA is underestimated and underdiagnosed.

A sleep history, simple upper airway examination, and quick validated screening tool like the STOP-BANG or Epworth Sleepiness Scale aid in identifying the need for testing for OSA.

Polysomnogram is the gold standard for evaluation of OSA. Home sleep apnea tests can be used to confirm a diagnosis of OSA in patients at high risk for moderate to severe OSA.
Dr. Vensel Rundo reported no financial interests or relationships that pose a potential conflict of interest with this article.

This article is based on Dr. Vensel Rundo's "Obstructive Sleep Apnea Basics" webcast released January 30, 2019, part of the "Obstructive Sleep Apnea: A Cleveland Clinic State-of-the-Art Review" online series (available at www.clevelandclinicmeded.com/online/sleep-apnea/default.asp). The article was drafted by Cleveland Clinic Journal of Medicine staff and was then reviewed, revised, and approved by Dr. Vensel Rundo.

doi:10.3949/ccjm.86.s1.02

\section{DEFINITION}

Obstructive sleep apnea (OSA) occurs when there are recurrent episodes of upper airway collapse and obstruction during sleep associated with arousals with or without oxygen desaturations. The oropharynx in the back of the throat collapses during OSA events to cause arousal or oxygen desaturation or both resulting in fragmented sleep.

\section{PREVALENCE}

Studies reveal OSA is prevalent. A 2015 study in Switzerland reported $50 \%$ of men and $23 \%$ of women had at least moderate OSA. ${ }^{1}$ In 2002, the Sleep Heart Health study found that 24\% of men and 9\% of women have at least mild OSA. ${ }^{2}$ In the Wisconsin Sleep Study Cohort, it was reported that $10 \%$ of men and $3 \%$ of women age 30 to 49 have at least moderate OSA, while $17 \%$ of men and $9 \%$ of women age 50 to 70 have at least moderate OSA. ${ }^{3}$ OSA is highly underrecognized and it is estimated that $82 \%$ of men and $93 \%$ of women in the United States with OSA are undiagnosed. ${ }^{4}$

\section{SYMPTOMS}

There are several common sleep and daytime symptoms associated with OSA though patients vary in the number and combination of symptoms reported (Table 1). ${ }^{5}$ During sleep, snoring is one of the most common symptoms. Common daytime symptoms of OSA include excessive daytime sleepiness or fatigue. Excessive daytime sleepiness is feeling very drowsy or very sleepy at times whereas fatigue is feeling tired, low on energy, and unmotivated. Feeling unrefreshed despite getting the recommended 7 to 9 hours of sleep is also a symptom.

\section{RISK FACTORS}

The risk of OSA is influenced by unmodifiable and modifiable factors. Unmodifiable risk factors include male sex, age, and race. Genetic predisposition or a family history of OSA as well as cranial facial anatomy resulting in narrow airways may impart higher 
risk of OSA. Modifiable risk factors include obesity, medications that cause muscle relaxation and narrowing of the airway (opiates, benzodiazepines, alcohol), endocrine disorders (hypothyroidism, polycystic ovarian syndrome), smoking, and nasal congestion or obstruction. $^{6}$

\section{Sex}

Men are at higher risk for OSA than women although once women reach menopause they have a risk similar to men. Postmenopausal women on hormone replacement therapy were found to have lower rates of OSA, suggesting that loss of hormones results in greater risk of OSA. ${ }^{7,8}$ Women also have more OSA during rapid eye movement (REM) sleep and less OSA when sleeping supine, whereas most men have OSA when sleeping supine. ${ }^{9,10}$ OSA is less severe in women compared with men of similar body mass index (BMI). ${ }^{11}$ Symptoms vary in men and women: snoring and witnessed apneas are more common in men whereas insomnia and excessive daytime sleepiness are more common in women. ${ }^{11}$ This may account for delayed diagnosis and the higher mortality in women compared with men.

\section{Age}

The risk of OSA increases with age. In a study of men 65 or older, the prevalence of moderate OSA was $23 \%$ in men younger than 72 and $30 \%$ in men older than $80 .{ }^{12}$ By comparison, the prevalence of moderate

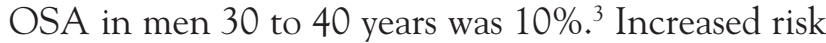
of OSA with age may be due to age-related reduction in slow wave sleep (ie, deep sleep), which is protective against sleep-disordered breathing and airway collapse. ${ }^{13}$ Older adults are also less symptomatic, reporting less daytime sleepiness and fatigue. ${ }^{14}$

\section{Race}

The Sleep Heart Health Study found a slightly increased risk of moderate to severe OSA in blacks (20\%) and American Indians (23\%) compared with whites (17\%). ${ }^{2}$ Another study showed the prevalence of OSA was 30\% in whites, $32 \%$ in blacks, $38 \%$ in Hispanics, and 39\% in Chinese individuals. ${ }^{15}$ A higher prevalence of OSA in young blacks $(\leq 25$ years) compared with whites was reported, ${ }^{16}$ although another study found no differences based on race in older patients. ${ }^{17}$ These differences among racial groups may be due to variations in craniofacial anatomy.

\section{Obesity}

There is a correlation between increased risk of OSA and obesity (BMI $\left.>30 \mathrm{~kg} / \mathrm{m}^{2}\right)$ and its correlates of greater waist-to-hip ratio and neck circumference. ${ }^{2}$

\begin{tabular}{ll} 
TABLE 1 & \\
Obstructive sleep apnea symptoms \\
\hline Sleep & Daytime \\
\hline Snoring & Excessive sleepiness \\
Gasping & Fatigue \\
Snorting & Morning headaches \\
Not breathing & Memory or concentration issues \\
$\begin{array}{l}\text { Fragmented sleep } \\
\text { Sleep maintenance } \\
\text { insomnia (inability to stay }\end{array}$ & Mood disturbances or irritability \\
$\begin{array}{l}\text { asleep or waking and not } \\
\text { returning to sleep) }\end{array}$ & \\
Nocturia & \\
Bedwetting & \\
Night sweats &
\end{tabular}

Data from reference 5 .

A $10 \%$ increase in body weight results in a sixfold increase in moderate to severe OSA and increases the apnea-hypopnea index (AHI; number of breath pauses or respiratory events per hour) by $32 \%$ whereas a $10 \%$ decrease in weight decreases the AHI by $26 \% .^{18}$

\section{COMORBIDITIES}

OSA is associated with a number of comorbid conditions including stroke, myocardial infarction, hypertension, hyperlipidemia, glucose intolerance, diabetes, arrhythmias including atrial fibrillation, pulmonary hypertension, congestive heart failure, and depression. Patients with moderate or severe OSA are at higher risk of these comorbid conditions. ${ }^{19}$

Patients with cardiovascular disease have a very high prevalence of OSA: hypertension ( $83 \%$ mild to $30 \%$ moderate to severe OSA), heart failure $(55 \%$ to $12 \%$ ), arrhythmias ( $50 \%$ to $20 \%$ ), stroke ( $75 \%$ to $57 \%)$, and coronary heart disease (65\% to $38 \%) .{ }^{20}$ Increased awareness and early diagnosis of OSA is critical to reducing cardiovascular disease burden.

\section{SCREENING}

Screening patients for OSA starts with a good sleep history to identify symptoms, risk factors, and comorbid conditions, as well as a physical examination for OSA-related features (Table 2). The Epworth Sleepiness Scale and STOP-BANG questionnaire are brief, effective screening tools that can inform the need for further testing. 
TABLE 2

Screening for asymptomatic populations

- Sleep history

- Epworth Sleepiness Scale

- Upper airway examination

- STOP-BANG

\section{Sleep history}

A sleep history starts with determining the patient's total sleep time, based on time to bed, time to fall asleep, and time of wake up, including any difficulty falling asleep, staying asleep, or daytime naps.

Symptoms. Daytime naps generally indicate a sleep deficit or sleep that is not refreshing. A review of sleep and daytime symptoms associated with OSA (Table 1) helps determine if excessive daytime sleepiness or unrefreshing sleep is out of proportion with the amount of sleep the patient is getting at night.

Some patients with OSA may have memory or concentration issues or feel like they have attention deficit disorder. In fact some patients are diagnosed with attention deficit disorder because of their insufficient sleep or unrefreshing sleep.

Drowsy driving is a special concern in patients with untreated OSA and sleep deprivation. Many patients have drowsy driving episodes or difficulty staying awake during long-distance driving. Caffeine use is also important information as excessive caffeine may be used to combat sleepiness during the day.

The Epworth Sleepiness Scale is a clinical screening tool that presents 8 situations for patients to consider and indicate their level of sleepiness and likelihood of falling asleep (never $=0$; slight $=1$; moderate $=2$, high $=3)^{21,22} \mathrm{~A}$ total score $\geq 10$ is considered abnormal in that the patient is excessively sleepy compared with most people.

Risk factors and comorbid conditions. OSA risk factors and comorbidities, including a BMI obesity assessment, should be reviewed with patients. Nasal congestion or mouth breathing especially at night could be due to airway obstruction increasing the risk of OSA. Family history of OSA, tobacco, alcohol use, other medical conditions, and medications should also be discussed.

\section{Physical examination}

Certain findings on physical examination could suggest the presence of OSA:

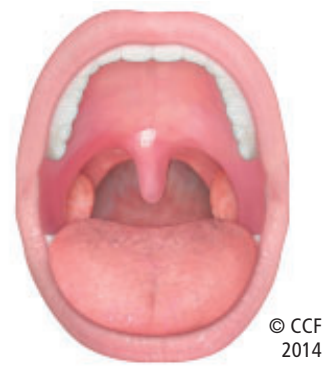

Class 1: soft palate, fauces, uvula, anterior and posterior pillars visible

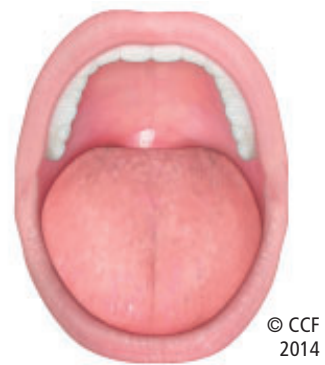

Class 3: soft palate and base of uvula visible

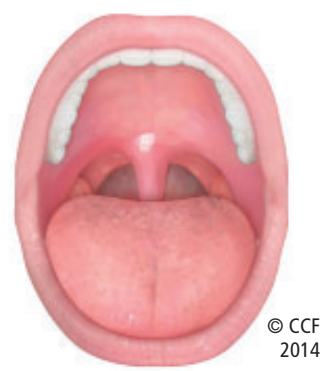

Class 2: soft palate, fauces and uvula visible

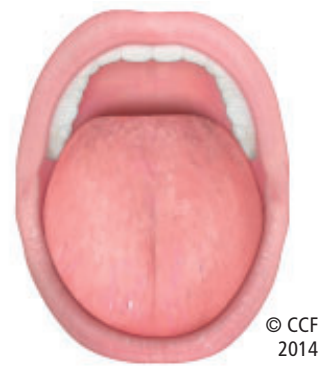

Class 4: soft palate not visible
Figure 1. Friedman palate positions (classes 1, 2, 3, and 4).

- Neck circumference greater than 17 inches for men or greater than 16 inches for women

- BMI greater than 30

- Friedman class tongue position class 3 or greater (Figure 1)

- Mouth features (present/enlarged tonsils, macroglossia, jaw misalignment)

- Nasal abnormalities (turbinate hypertrophy, deviated septum). ${ }^{5}$

Patients with Friedman palate positions class 3 and 4 have a higher risk of OSA due to airway crowding during sleep when the airway naturally collapses a little and is even more restricted.

Narrow airways or oropharyngeal crowding can also be due to a swollen, enlarged, or elongated uvula; present or enlarged tonsils; or lateral wall narrowing. Alone or in combination, these features can contribute to airway obstruction.

Other signs in the mouth suggestive of obstruction are macroglossia (enlarged tongue) and tongue ridging. Tongue ridging or scalloping impressions typically occur during sleep and are caused by the tongue moving forward to open the airway and pressing against the teeth. 
Normal sleep

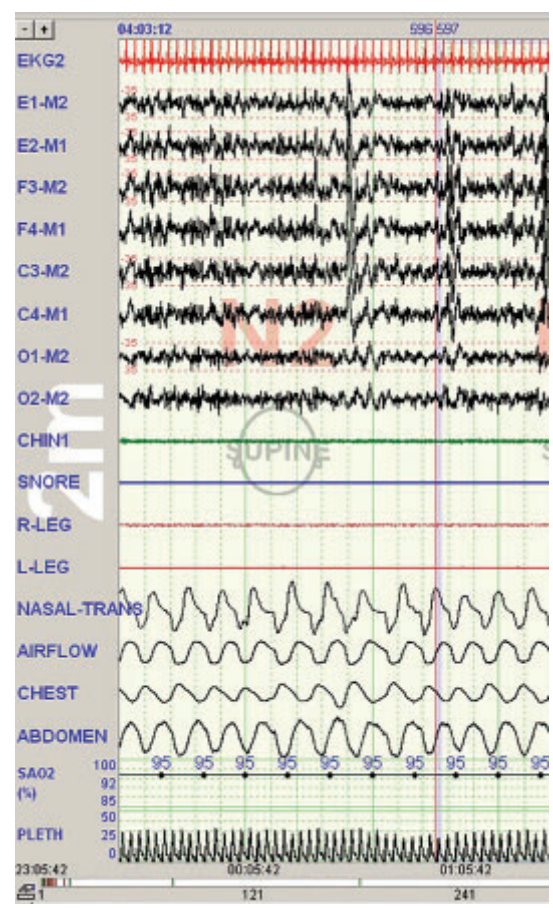

Waveform patterns typical of sleep.

\section{Obstructive hypopnea}

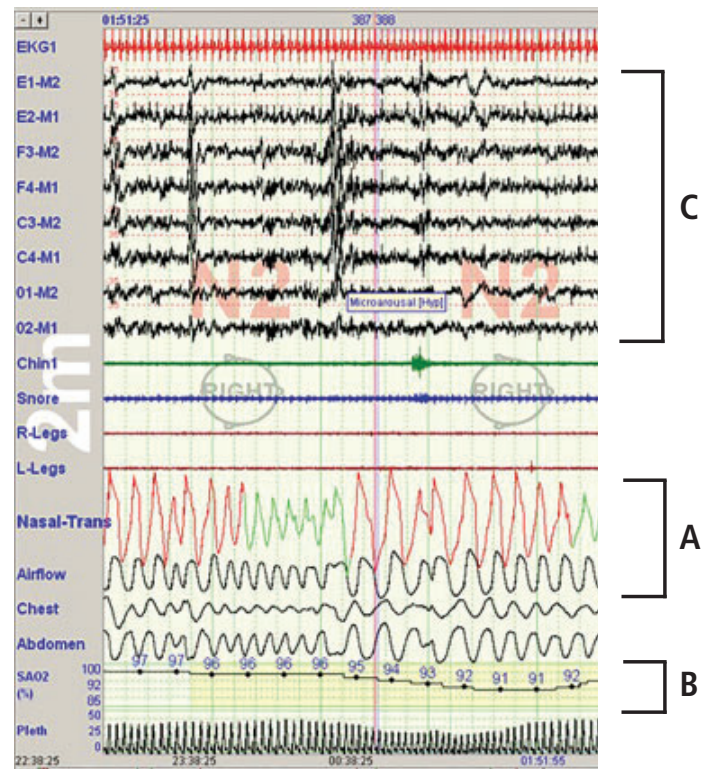

Reduced flow in the nasal transducer and airflow leads (A) with increased oxygen desaturation (B) and arousal (C).
Obstructive apnea

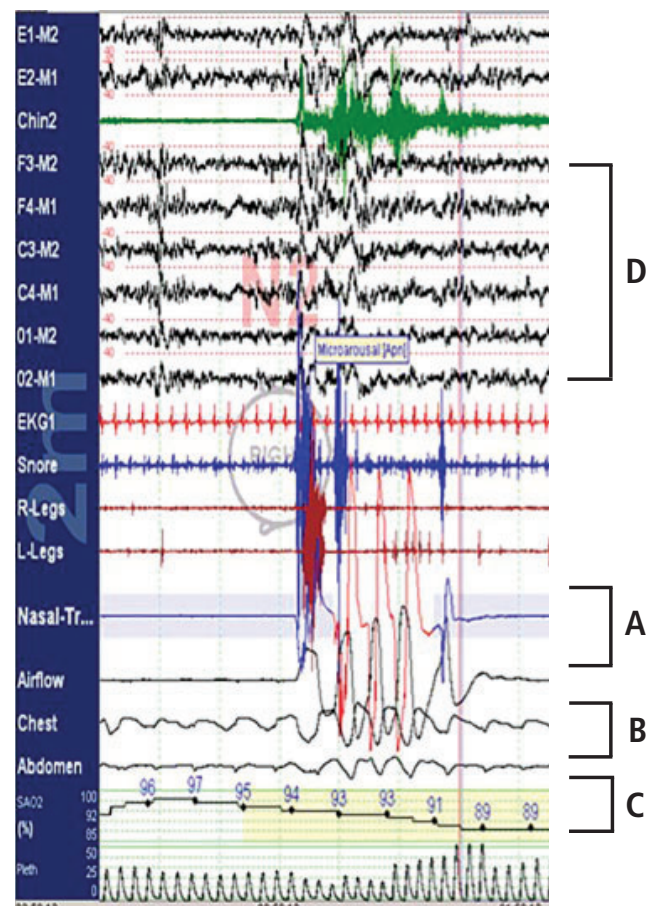

Airflow in the nasal transducer and the airflow leads (A) show no airflow but there is persistent effort in the chest and abdomen leads (B). Oxygen desaturation (C) and arousal in the electroencephalogram leads (D) also occurs in association with this respiratory event.

\section{Respiratory event-related arousal}

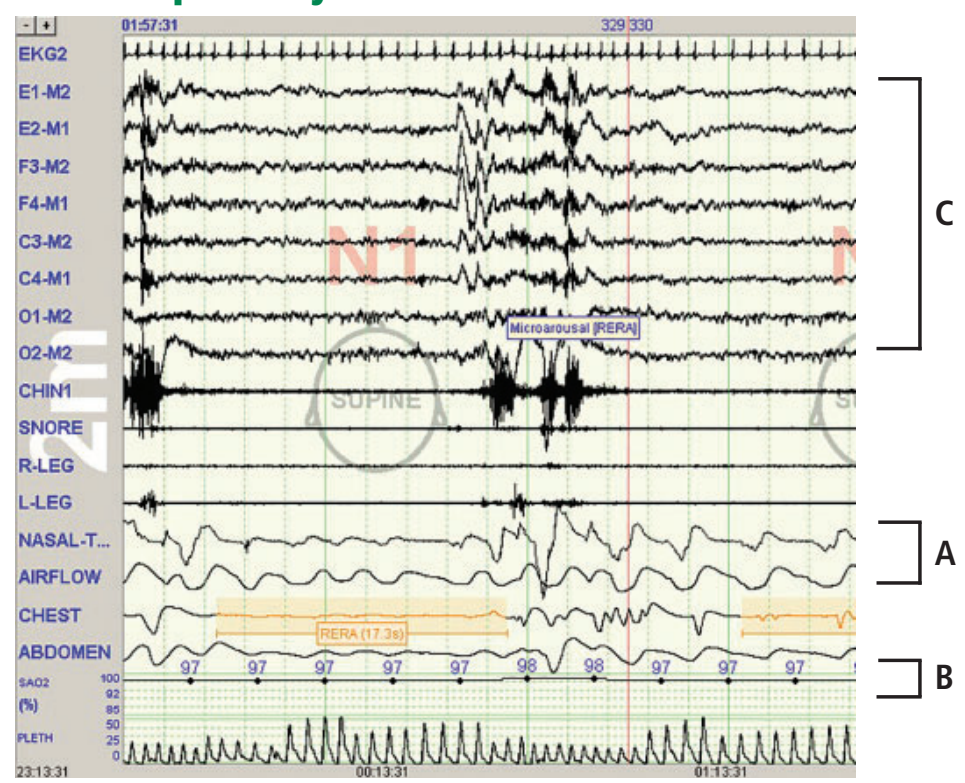

Reduction in airflow in the nasal transducer and airflow leads (A) and slight oxygen desaturation (B) and arousal from sleep in the electroencephalogram leads (C).

Figure 2. Polysomnogram excerpts with normal sleep, obstructive apnea, obstructive hypopnea, and respiratory event-related arousal waveform findings. 
TABLE 3

Polysomnogram parameters and associated leads

\begin{tabular}{ll} 
Parameter & Lead(s) \\
\hline $\begin{array}{l}\text { Heart rate and rhythm (EKG/ECG) } \\
\text { Brain waves (EEG) }\end{array}$ & EKG2 \\
& F3-M2, F4-M1, C3-M2, C4-M1, 01-M2, \\
O2-M2 & E1-M2 and E2-M1 \\
Eye movement (EOG) & CHIN1, R-LEG, L-LEG \\
Awake/asleep, REM sleep, chin and & NASAL-TRANS, AIRFLOW \\
leg movement (EMG) & CHEST, ABDOMEN \\
Nasal-oral airflow & SAO 2 \\
Thoraco-abdominal effort & SNORE \\
Oxygen saturation & SUPINE (watermark) \\
Snore & \\
Body position &
\end{tabular}

$\mathrm{EEG}=$ electroencephalogram; EKG/ECG = electrocardiogram; EMG = electromyogram; EOG = electrooculography; $R E M=$ rapid eye movement

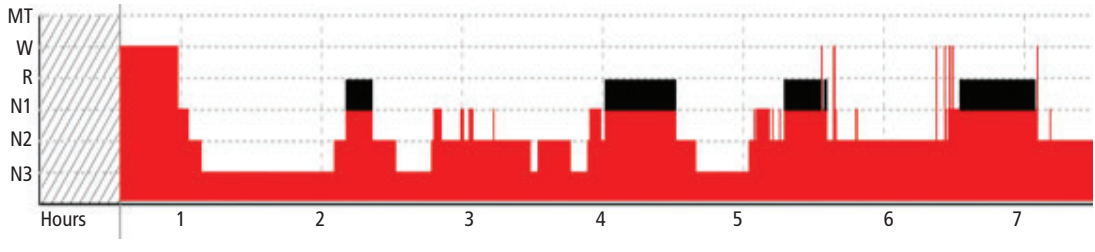

$\mathrm{MT}=$ monitoring time; $\mathrm{N} 1=$ stage $1 ; \mathrm{N} 2=$ stage $2 ; \mathrm{N} 3=$ stage $3 ; \mathrm{R}=\mathrm{REM}$ sleep $($ black); $\mathrm{W}=$ wake

Figure 3. A hypnogram depicts the recorded levels of sleep over time.

Retrognathia (lower jaw offset behind upper jaw) can narrow the airway and increase the risk of OSA as can a high arch palate, overbite (upper teeth forward), or overjet (upper teeth over the top of lower teeth).

A nasal examination for nasal valve collapse (ie, nostril collapses with inhalation), deviated septum, and inferior turbinate hypertrophy impart an increased risk of OSA.

\section{Screening tools}

In addition to the Epworth Sleepiness Scale, the STOP-BANG questionnaire can help determine if a patient should be tested further for OSA. The STOPBANG questionnaire consists of 8 yes-no questions where more than 2 yes responses indicate the patient is at higher risk for moderate to severe OSA (93\% sensitivity): Snore, Tired, Observed stopped breathing, high blood Pressure, BMI $>35 \mathrm{~kg} / \mathrm{m}^{2}$, Age $>50$, Neck $>15.75$ inches, Gender $=$ male $).{ }^{23}$

\section{SLEEP STUDIES}

Polysomnography (PSG) is the gold standard of evaluation for OSA. The more recently availabile home sleep apnea test (HSAT) is convenient for select patients as a confirmatory test but results may underestimate the severity of sleep-related breathing disorders.

\section{Polysomnography}

PSG is a monitored, 8-hour sleep study conducted in a laboratory with an established scoring criteria for OSA-related respiratory events. ${ }^{24}$ The test can be tailored to a patient's clinical history to determine the need for supplemental oxygen and positive airway pressure titration, detect elevated carbon dioxide (hypercapnia or hypoventilation) due to shallow breathing, and monitor for seizures or parasomnias. The PSG also records REM and nonREM sleep for REMrelated sleep disorders, body position (supine and off supine), and variability in muscle tone that corresponds to the different stages of sleep (Figure 2, Table 3).

Hypnogram. A hypnogram is a type of polysomnography that illustrates the different stages of sleep over time: wake, stage 1 , stage 2 , and stage 3 , and REM sleep (Figure 3). In a typical night's sleep of 7 to 9 hours, patients cycle through the sleep stages 4 to 5 times. A hypnogram can also include waveforms for other parameters such as body position, respiratory events (apnea and hypopneas), microarousals, continuous positive airway pressure therapy, and oxygen saturation.

\section{Home sleep apnea test}

HSATs record 4 to 7 parameters including airflow (thermal and nasal pressure), effort (inductive plethysmography), and oximetry. No electroencephalogram is used, so sleep is not recorded; it is assumed the patient is sleeping for the duration of the test. As such, respiratory events are based on oxygen desaturations and reduced airflow and pressure as well as chest and abdomen effort. The raw data are edited and manually scored and reviewed by a sleep specialist. ${ }^{25}$

Although the HSAT is convenient for many 
patients, it underestimates the severity of sleep-related breathing disorders. HSAT is intended to confirm OSA in patients with a high likelihood of OSA based on their sleep history. ${ }^{26} \mathrm{It}$ is ideally employed for adult patients with no major medical problems or other sleep problems who are at high risk for moderate to severe OSA based on the STOP-BANG questionnaire or those with daytime sleepiness and 2 of the 3 symptoms of snoring, witnessed apnea, or hypertension. ${ }^{27}$

A negative or inconclusive HSAT warrants a PSG to ensure the patient does not have OSA. Use of HSAT is contraindicated in patients with

- Significant cardiopulmonary disease

- Potential weakness due to a neuromuscular condition

- Awake hypoventilation or high risk for sleep-related hypoventilation (severe obesity)

- History of stroke

- Chronic opioid use

- Severe insomnia

- Symptoms of other significant sleep disorders

- Environmental/personal factors that would preclude adequate acquisition and interpretation of data (disruptions from children, pets, other factors)..$^{27}$

\section{DIAGNOSTIC CRITERIA}

Results from a PSG or HSAT inform the diagnosis of OSA and the need for treatment. The current diagnostic criteria for OSA were established in 2014 by the American Academy of Sleep Medicine (Table 4). ${ }^{28}$

\section{Respiratory events captured on a PSG or HSAT}

The OSA diagnostic criteria are based on the occurrence of obstructive respiratory events recorded during sleep such as apneas, hypopneas, and respiratory event-related arousals.

Apneas. An apnea is a respiratory event resulting in a complete lack of airflow as measured by a greater than $90 \%$ reduction in thermal sensor for 10 or more seconds. Apneas can be obstructive, central, or mixed (Figure 4). Obstructive apneas occur when the airway is closed and respiratory effort is present in the chest

TABLE 4 ollowing:
Polysomnogram or home sleep apnea test reveals

- $\geq 15$ predominantly obstructive respiratory events per hour of sleep OR

- $\geq 5$ predominantly obstructive respiratory events per hour of sleep and at least 1 of

- Hypertension, mood disorder, cognitive dysfunction, ischemic heart disease, stroke, congestive heart failure, atrial fibrillation, or diabetes type 2

Data from reference 28

\section{Obstructive \\ (no airflow \\ with respiratory effort)}

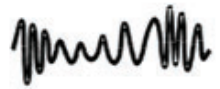

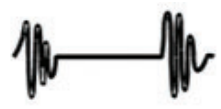

Mixed (no airflow with and without respiratory effort)
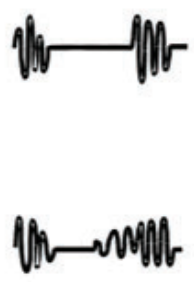

Central (no airflow and no respiratory effort)
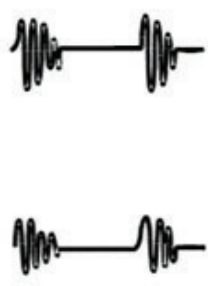

Figure 4. Apneas can be obstructive, mixed, or central.

and abdomen (Figure 2). In central apnea, there is no airflow and no respiratory effort, meaning the brain is not directing the body to breathe. Mixed apneas cause a lack of airflow with and without respiratory effort.

Hypopneas. A hypopnea is a respiratory event resulting in reduced airflow. The America Association of Sleep Medicine's preferred definition is a reduction in nasal pressure of at least $30 \%$ for 10 seconds or longer with $3 \%$ or greater oxygen desaturation or an electroencephalogram arousal. Another acceptable definition is at least $30 \%$ reduction in thoracoabdominal movement or airflow with $4 \%$ or greater oxygen desaturation, which is used by the Centers for Medicare and Medicaid Services and other insurers. ${ }^{29,30}$ Hypopnea requires greater oxygen desaturation and is not dependent on arousals, which can sometimes make it more challenging to identify OSA (Figure 2).

Respiratory event-related arousals. Respiratory event-related arousals are respiratory events not meeting apnea or hypopnea criteria. They are measured 
TABLE 5

Obstructive sleep apnea severity

\begin{tabular}{ll}
\hline Index & Calculation \\
\hline Apnea-hypopnea index (AHI) & $\frac{\text { Apneas + Hypopneas }}{\text { Total sleep time, hours }}$ \\
Respiratory disturbance index & $\frac{\text { AHI + Respiratory event-related arousals }}{\text { Total sleep time, hours }}$ \\
Respiratory event index & $\frac{\text { Apneas + Hypopneas }}{\text { Total monitoring time, hours }}$
\end{tabular}

Respiratory event index is typically used for home sleep apnea testing as it is based on monitoring time as distinct from actual sleep time.

2. Young T, Shahar E, Nieto FJ, et al; for the Sleep Heart Health Study Research Group. Predictors of sleep-disordered breathing in community-dwelling adults. Arch Intern Med 2002; 162(8):893-900.

3. Peppard PE, Young T, Barnet JH, Palta M, Hagen EW, Hla KM. Increased prevalence of sleep-disordered breathing in adults. Am J Epidemiol 2013; 177(9):1006-1014.

4. Young T, Evans L, Finn L, Palta M. Estimation of clinically diagnosed proportion of sleep apnea syndrome in middle-aged men and women. Sleep 1997; 20(9):705-706.

5. Epstein U, Kristo D, Strollo Jr, PJ, et al; Adult Obstructive Sleep Apnea Task Force of the American Academy of Sleep Medicine. Clinical guideline for the evaluation, management and longterm care of obstructive sleep apnea in adults. J Clin Sleep Med 2009; 5(3):263-276.

6. Young T, Skatrud J, Peppard PE. Risk factors for obstructive sleep apnea in adults. JAMA 2004; 291(16):2013-2016.

7. Young T, Finn L, Austin D, Peterson A. Menopausal status and sleep-disordered breathing in

as a sequence of breaths of 10 or more seconds with increasing respiratory effort or flattening of the nasal pressure waveform leading to arousal (Figure 2). ${ }^{29}$ Respiratory event-related arousals are disruptive to sleep and have many of the same consequences as apneas and hypopneas.

\section{Severity}

A diagnosis of OSA should include a measure of severity (mild, moderate, or severe) as the severity may determine if a patient with OSA is treated or not. Severity is determined by AHI, respiratory disturbance index, or respiratory event index (Table 5). ${ }^{29}$ For any of the 3 indexes, a value 5 to 14.9 is considered mild, 15 to 29.9 is considered moderate, and 30 or greater is considered severe.

\section{SUMMARY}

OSA results from airway collapse and obstruction during sleep, often causing arousal from sleep with or without oxygen desaturation. The prevalence of OSA is underestimated and it is underdiagnosed despite known risk factors and comorbid conditions. Screening for OSA with a sleep history, simple upper airway examination, and quick validated screening tool like the STOP-BANG or Epworth Sleepiness Scale aid in identifying the need for testing for OSA. A laboratory sleep study with a PSG can confirm the diagnosis and severity of OSA. HSATs are available to confirm the diagnosis of OSA in patients at high risk for moderate to severe OSA.

\section{REFERENCES}

1. Heinzer R, Vat S, Marques-Vidal P, et al. Prevalence of sleep-disordered breathing in the general population: the HypnoLaus study. Lancet Respir Med 2015; 3(4):310-318. the Wisconsin Sleep Cohort Study. Am J Respir Crit Care Med 2003; 167(9):1181-1185.

8. Shahar E, Redline S, Young T, et al; for the Sleep Heart Health Study Research Group. Hormone replacement therapy and sleep-disordered breathing. Am J Respir Crit Care Med 2003; 167(9):1186-1192.

9. O'Connor C, Thornley KS, Hanly PJ. Gender differences in the polysomnographic features of obstructive sleep apnea. Am J Respir Crit Care Med 2000; 161(5):1465-1472.

10. Collop NA, Adkins D, Phillips BA. Gender differences in sleep and sleep-disordered breathing. Clin Chest Med 2004; 25(2):257-268.

11. Redline S, Kump K, Tishler PV, Browner I, Ferrette V. Gender differences in sleep disordered breathing in a community-based sample. Am J Respir Crit Care Med 1994; 149(3 Pt 1):722-726.

12. Mehra R, Stone KL, Blackwell T, et al; for the Osteoporotic Fractures in Men Study. Prevalence and correlates of sleep-disordered breathing in older men: Osteoporotic Fractures in Men Sleep Study. J Am Geriatr Soc 2007; 55(9):1356-1364.

13. Van Cauter E, Leproult R, Plat L. Age-related changes in slow wave sleep and REM sleep and relationship with growth hormone and cortisol levels in healthy men. JAMA 2000; 284(7):861-868.

14. Groth M. Sleep apnea in the elderly. Clin Geriatr Med 2005; 21:701-712.

15. Chen X, Wang R, Zee $\mathbf{P}$, et al. Racial/ethnic differences in sleep disturbances: the Multi-Ethnic Study of Atherosclerosis (MESA). Sleep 2015; 38(6):877-888.

16. Redline S, Tishler PV, Hans MG, Tosteson TD, Strohl KP, Spry K. Racial differences in sleep-disordered breathing in African-Americans and Caucasians. Am J Respir Crit Care Med 1997; 155(1):186-192.

17. Song Y, Ancoli-Israel S, Lewis CE, Redline S, Harrison SL, Stone KL. The association of race/ethnicity with objectively measured sleep characteristics in older men. Behav Sleep Med 2011; 10(1):54-69.

18. Peppard PE, Young T, Palta M, Dempsey J, Skatrud J. Longitudinal study of moderate weight change and sleep-disordered breathing. JAMA 2000; 284(23):3015-3021.

19. Shahar E, Whitney CW, Redline S, et al. Sleep-disordered breathing and cardiovascular disease: cross-sectional results of the Sleep Heart Health Study. Am J Respir Crit Care Med. 2001;163:19-25

20. Javaheri S, Barbe F, Campos-Rodriguez F, et al. Sleep apnea: types, mechanisms, and clinical cardiovascular consequences. J Am Coll Cardiol 2017; 69(7):841-858.

21. Johns MW. Daytime sleepiness, snoring, and obstructive sleep apnea. Chest 1993; 103(1):30-36.

22. Chervin RD, Aldrich MS. The Epworth Sleepiness Scale may not reflect objective measures of sleepiness or sleep apnea. Neurology 1999; 52(1):125-131.

23. Chung F, Yegneswaran B, Liao P, et al. STOP questionnaire: a tool to screen patients for obstructive sleep apnea. Anesthesiology 2008; 108(5):812-821. 
24. Iber C, Ancoli-Israel S, Chesson A, Quan SF; for the American Academy of Sleep and Medicine. The ASSM Manual for the Scoring of Sleep and Associated Events: Rules, Terminology and Technical Specifications. 1st ed. Winchester, IL: American Academy of Sleep Medicine; 2007.

25. Centers for Medicare and Medicaid Services. Medicare Learning Network. Continuous positive airway pressure (CPAP) therapy for obstructive sleep apnea (OSA). www.cms.gov/Outreach-andEducation/Medicare-Learning-Network-MLN/MLNMattersArticles/ downloads/mm6048.pdf. Accessed August 19, 2019.

26. Collop NA, Anderson WM, Boehlecke B, et al; Portable Monitoring Task Force of the American Academy of Sleep Medicine. Clinical guidelines for the use of unattended portable monitors in the diagnosis of obstructive sleep apnea in adult patients. J Clin Sleep Med 2007; 3(7):737-747.

27. Kapur VK, Auckley DH, Chowdhuri S, et al. Clinical practice guide- line for diagnostic testing for adult obstructive sleep apnea: an American Academy of Sleep Medicine clinical practice guideline. J Clin Sleep Med 2017; 13(3):479-504.

28. Sateia MJ. International classification of sleep disorders-3rd ed: highlights and modifications. Chest 2014; 146(5):1387-1394.

29. AASM Manual for the Scoring of Sleep and Associated Events: Rules, Terminology and Technical Specifications. Version 2.5. American Academy of Sleep Medicine; April 2018.

30. Centers for Medicare and Medicaid Services. Medicare Coverage Database. www.cms.gov/medicare-coverage-database. Accessed August 19, 2019.

Correspondence: Jessica Vensel Rundo, MD, MS, Sleep Disorders Center, S73, Cleveland Clinic, 9500 Euclid Avenue, Cleveland, OH 44195; venselj@ccf.org 